\title{
Recognition failure of words with a single meaning
}

\author{
ENDEL TULVING and OLGA C. WATKINS \\ University of Toronto, Toronto, Canada MSS IAI
}

\begin{abstract}
Recognition failure of recallable words was demonstrated with single-meaning target words after a 7-day retention interval. When overall levels of recognition were equated, the magnitude of the effect was the same as that observed with high-frequency words of multiple meanings in other experiments. It was concluded that, contrary to suggestions of Reder, Anderson, and Bjork (1974), encoding specificity is not limited to words that have several semantic senses. The experiment also provided evidence contrary to the hypothesis that recall of unrecognized items comes about because of additional learning opportunities afforded by the recognition test.
\end{abstract}

Under certain conditions people cannot recognize previously studied words that they can recall. These conditions are defined by the features of what is called the recognition-failure paradigm, as follows: (1) Subjects study pairs of words (A-B), (2) their recognition memory for $B$ members of pairs is tested, with extralist words as lures, and (3) recall of $B$ members of pairs is tested with $\mathrm{A}$ members as retrieval cues. The phenomenon of recognition failure represents, at the empirical level, a particularly clear demonstration of context effects in episodic memory and, at the theoretical level, a special case of encoding specificity (Tulving \& Thomson, 1973).

This article describes the results of an experiment that are relevant to the issue of the generality of the phenomenon of recognition failure of recallable words (or recognition failure). Existing evidence shows that whenever the standard paradigm has been used, recognition failure has been observed (Tulving \& Wiseman, 1975; Wiseman \& Tulving, 1976). Some investigators (e.g., Postman, 1975; Reder, Anderson, \& Bjork, 1974; Salzberg, 1976) have suggested limits to the generality of encoding specificity based on the observation that sometimes recognition is higher than recall in the standard paradigm. Wiseman and Tulving (1976) have argued, however, that the comparison of overall recognition and recall rates is not quite appropriate, since recognition failure can, and usually does, occur in experiments in which recognition exceeds recall. The data reported by Postman (1975) and Salzberg (1976) do in fact show recognition failure, and the data collected by Reder et al. (1974), when reanalyzed by Tulving and Wiseman (1975), were also found to reveal recognition failure. Moreover, the relation between

This research has been supported by the National Research Council of Canada, Grant A8632. Requests for reprints should be sent to E. Tulving, Department of Psychology, University of Toronto, Toronto, Canada M5S 1A1. Olga C. Watkins is now at Princeton University. We are grateful to Michael Watkins for help with the construction of experimental materials. the magnitude of recognition failure and overall recognition in all these experiments, as well as a number of others (e.g., Tulving, 1974; Tulving \& Thomson, 1973; Watkins \& Tulving, 1975; Wiseman \& Tulving, 1975) has been found to be highly systematic and largely invariant with characteristics of materials and procedural details used.

The primary question to which the experiment reported in the present article was directed was this: Does recognition failure occur with words that have only a single meaning? The motivation for posing this question was provided by an explanation of encoding specificity suggested by Reder et al. (1974). These authors assumed that studying a list word involves associating information about the word's occurrence in the list to its representation in (permanent) memory. This occurrence information must be accessible for recall or recognition to be successful at the time of the test. Most words, particularly high-frequency words, have several meanings or semantic senses, and hence several representations in semantic memory, only one or some of which may be encoded at the time of the study, that is, tagged with appropriate occurrence information. Reder et al. proposed that the senses tagged at study and the senses consulted for occurrence information at the time of test are determined, among other things, by the context in which study and test occur. Recognition failure of a recallable word occurs if, because of different contexts, untagged senses of the target word are (implicitly) retrieved in the recognition test, and tagged senses in the cued recall test.

Although Reder et al. did not report any data on the extent to which recallable words in their experiment were not recognized, their results did show that for the high-frequency target words recall was higher than recognition, while for low-frequency target words this relation between recall and recognition was reversed. They concluded that "low-frequency words did not support the Encoding Specificity Principle" (Reder et al., 1974, p. 648). 
The results and the conclusion of Reder et al. have been widely accepted as imposing an important limitation on the generality of the phenomenon of recognition failure and hence on its theoretical implications. Thus, for instance, Postman (1975) has suggested that "apparent manifestations of encoding specificity" occur only under "narrowly circumscribed conditions," and mentioned the Reder et al. (1974) experiment as an illustration of such conditions. Baddeley (1976) described the Reder et al. experiment as showing that "the encoding specificity effect occurred only in the case of high-frequency words" (p. 296). Miller and Johnson-Laird (1976) also took the Reder et al. results to mean that the principle of encoding specificity "does not hold for rare words, which have few senses" (p. 156).

Because of the clear significance of the results of the Reder et al. (1974) study and their theoretical implications, the issue of recognition failure and multiplicity of word meanings in semantic memory seemed worthy of further examination. Tulving and Wiseman (1975) reanalyzed the Reder et al. data and found that both high- and low-frequency words exhibited recognition failure, in line with the results of other experiments. But it is possible that this outcome reflects only the less-than-perfect correlation between word frequency and number of semantic senses, and that even the low-frequency words in the Reder et al. study showed recognition failure because at least some of them in fact had multiple meanings. In the experiment to be described here, only those words were selected as materials for the experiment that clearly had only a single meaning. Recognition failure of these words would extend the generality of the phenomenon; it would also make it difficult to retain the argument that encoding specificity depends on multiple semantic senses of words in the subject's preexperimentally established repertoire.

A second issue dealt with in the present article has to do with the possibility that recognition failure is either wholly or in part an artifact of the procedure used in experiments demonstrating it. In this procedure, the subject's knowledge for list items is tested twice in succession, first in a recognition test, and then in a cued recall test. It is possible that subsequent recall of initially unrecognized words results from learning that occurs during the recognition test. Even if the subject does not recognize a test item, he may be able to remember it from the test and reproduce it later on, in the cued recall test, leading the experimenter to (mistakenly) conclude that the recalled item was learned in the original study list. The literature on the effects of a recognition test on subsequent recall (e.g., Cooper \& Monk, 1976; Postman, Jenkins, \& Postman, 1948; Slamecka, 1967) suggests that positive effects of one test on the other under the conditions of the recognition-failure paradigm cannot be ruled out. The present experimental design adds to the previous experiments concerned with this issue (Postman, 1975; Wiseman \& Tulving, 1975) the feature of an explicit recall test of items the subjects have seen only in the recognition test. Evidence about the extent to which subjects could make correct responses in the cued recall test only on the basis of having seen an item in the recognition test would be relevant to the evaluation of the hypothesis that recognition failure is an artifact of two successive retention tests.

\section{METHOD}

\section{Materials and Design}

A pool of 276 words was formed such that each word had, according to Webster's New World Dictionary, only a single meaning. The words were randomly assigned to two sets, with 180 words in one set and 96 in the other. The words in the smaller set were used as lures in the recognition tests. For each of the words in the larger set, a descriptive phrase was made up; each phrase was specific to its target word but unlikely to allow the word to be guessed. Examples are: a firm, friendly touch-HANDSHAKE; you can sometimes eat it, but never sit on it-CACTUS; a weapon that can be folded for storagePENKNIFE; holds a deadly piece of metal-HOLSTER; hairy on the outside but delicious on the inside-COCONUT; $a$ very busy biological matchmaker-ENZYME; found in certain colonies in old times-LEPROSY. Two sets of 18 phrase-word pairs were used as two versions of a set-establishing list. The remaining 144 phrase-word pairs were randomly divided into three sets of 48 pairs, serving as three different study lists, each used with one-third of the subjects.

All subjects were treated identically. Each subject first saw and was tested for cued recall of a set-establishing list. He was then presented the 48 phrase-word pairs of the critical list and given an immediate recognition test, followed by a cued recall test, of one-half of the targets. Seven days later, the subject returned for a second session in which he was tested for recognition and cued recall of the other half of the critical list in exactly the same way as in the immediate test.

The details of the design for the critical list are summarized in Table 1 . In the table, $A, B$, and $C$ represent three different sets of 48 phrase-word pairs, Subscripts 1 and 2 refer to randomly determined halves of each of these sets, and $X$ and $Y$ are symbols for two sets of 48 words used only as lures in the

Table 1

Design of Experiment

\begin{tabular}{|c|c|c|c|c|c|}
\hline \multirow{2}{*}{$\begin{array}{l}\text { Subject } \\
\text { Group }\end{array}$} & \multirow{2}{*}{$\begin{array}{c}\text { Study } \\
\text { List }\end{array}$} & \multicolumn{2}{|c|}{ Immediate Test } & \multicolumn{2}{|c|}{ Delayed Test } \\
\hline & & Recognition & Cued Recall & Recognition & Cued Recall \\
\hline $\begin{array}{l}1 \\
2 \\
3\end{array}$ & $\begin{array}{l}A_{1}+A_{2} \\
B_{1}+B_{2} \\
C_{1}+C_{2}\end{array}$ & $\begin{array}{l}A_{1}+\left(B_{1}+X\right) \\
B_{1}+\left(C_{1}+X\right) \\
C_{1}+\left(A_{1}+X\right)\end{array}$ & $\begin{array}{l}A_{1}+\left(B_{1}+C_{1}\right) \\
B_{1}+\left(C_{1}+A_{1}\right) \\
C_{1}+\left(A_{1}+B_{1}\right)\end{array}$ & $\begin{array}{l}A_{2}+\left(B_{2}+Y\right) \\
B_{1}+\left(C_{2}+Y\right) \\
C_{2}+\left(A_{2}+Y\right)\end{array}$ & $\begin{array}{l}A_{2}+\left(B_{2}+C_{2}\right) \\
B_{2}+\left(C_{2}+A_{2}\right) \\
C_{2}+\left(A_{2}+B_{2}\right)\end{array}$ \\
\hline
\end{tabular}

Note-Symbols in parentheses refer to sets of lures. 
recognition tests. For instance, the first subject group studied a list composed of items in Set $\mathrm{A}$ and was tested for retention of target words from Subset $A_{1}$ in the immediate test and words from Subset $A_{2}$ in the 7-day delayed test. In addition to 24 items from Subset $A_{1}$, the target items, there were 72 lures in the immediate recognition test: 24 words from Subset $B_{1}$ and 48 words from Subset $X$. In the cued recall test that followed the recognition test, subjects were provided with phrase cues for the 24 items in each of the three subsets, $A_{1}, B_{1}$, and $C_{1}$. Thus, in addition to providing information about the effectiveness of phrase cues for targets that the subjects had seen both in the study list and in the recognition test (Subset $A_{1}$ ), the cued recall test permitted us to observe the extent to which subjects recalled as list targets those words that they had seen only in the recognition test $\left(B_{1}\right)$ or had not seen at all in the experiment $\left(C_{1}\right)$. Furthermore, there were four successive stages in the cued recall test. First, the subjects indicated for each phrase whether they recognized it from the study list. They then wrote down opposite the appropriate phrases: (1) as many targets as they could remember from the study list, (2) words that they remembered from the recognition test only, and finally, (3) words that they did not remember appearing in either the study list or the recognition test but which seemed to fit some remaining phrases (i.e., guessing from semantic memory).

The sequence of events in the 7-day delayed test was exactly the same as in the immediate test, and the logic of the design was also exactly the same; the subjects now were trying to recall or guess the words from the complementary subsets of $\mathrm{A}, \mathrm{B}$, and $\mathrm{C}$.

Table 1 shows only one-half of the design; three other groups of subjects received the same study lists as the first three, but the sets of materials used in the immediate and delayed tests were reversed. The net result of this design was that the materials were completely balanced across all stages of the experiment.

\section{Subjects}

Twenty-four University of Toronto undergraduates took part in the experiment for pay. They were randomly assigned to six groups of four subjects; each group differed from the others only in the materials with which it was tested.

\section{Procedure}

The subjects were told that they would see a series of word lists on a closed-circuit television system and that their task was to try to remember the words. To help them, each word would be preceded by a phrase describing the to-be-remembered word. The phrases would be read aloud by the experimenter and later provided as cues for the subjects to recall the corresponding words. An example was given of a possible phrase-word pair.

Following these instructions, the set-establishing list of 18 phrase-word pairs was presented at the rate of approximately $8 \mathrm{sec} /$ pair. At the end of the presentation of this list, the subjects were given the same 18 phrases, in a new random order, and asked to try to recall the appropriate target words. The test was unpaced.

The subjects were then told that the next list would be a lot longer, but that their task was again to remember the target words in preparation for a cued recall test. The critical list of 48 phrase-word pairs was then presented, in the same way as the set-establishing lisi, and again at an 8 -sec rate. At the end of the presentation, the subjects were engaged in a digit-copying task for $1 \mathrm{~min}$, and were then given three successive tests of one-half of the phrase-word pairs. The first was a free-choice recognition test. It comprised four sheets of 24 words, 24 copies of target items and 72 lures as described in the Design section, and the subjects were asked to circle any and all words that they recognized as targets. They were given $5 \mathrm{~min}$ to complete this task.
Following the recognition test, the subjects were given a cued recall test in which they were provided with 72 phrases: 24 corresponding to study-list targets, 24 to recognition-lest lures, and 24 to items in the subjects' semantic memories only. The experimenter first told subjects to check the phrases that they remembered from the presentation list, and to place a cross beside those they thought were new. They were then asked to supply as many targets from the studied list as they could remember, writing each word beside its phrase cue. When all subjects had finished this recall task, they were given different colored pens, asked to look at all the phrases to which they had not responded, and to write down words fitting the given phrases that they remembered from the recognition test but had not yet reproduced as study-list items. After the second stage, the subjects were given a third pen of a different color and asked to go through the list of phrases once more and try to make up their own responses to phrases to which they had not yet responded. They were told that they did not have to supply a word to fit all of the remaining phrases, but only as many as they could. There was no time limit imposed on any of these tasks; most subjects spent a total of about $15 \mathrm{~min}$ completing the recall and guessing tasks.

The subjects returned after 1 week, ostensibly for a second experiment. After appropriate instructions, essentially identical to those they had received in the immediate test, they were tested on the other half of the critical list items in exactly the same way as they had been tested in the immediate test: (1) recognition of list words, (2) recognition of phrase cues, (3) cued recall of list words, (4) cued recall of recognition test items, and (5) guessing responses to cue phrases from semantic memory.

\section{RESULTS}

Immediate recall of set-establishing lists was essentially perfect: There were only three target words out of a total possible of 432 ( 24 subjects $\times 18$ items) that were not recalled. This extremely high level of recall can be interpreted as indicating the very high efficiency of the type of cues used in the experiment.

\section{Recognition and Recall of Study-List Targets}

A summary of the data pertaining to the fourfold classification of study-list targets in terms of their recognition and recall is presented in Table 2. Overall recognition hits and cued recall measures are provided along with data from the four cells of the 2 by 2 recognition/recall contingency table. The last two columns indicate the degree of recognition failure, one observed, calculated as a conditional probability of recognition miss given recall, and the other predicted on the basis of previous data (Tulving \& Wiseman, 1975). The data are shown both for the immediate and the 7-day delayed test, and data from the Reder et al. (1974) experiment are included for purposes of comparison. $^{1}$

Recognition hits were based on study-list target items that the subjects circled in the recognition test, and cued recall data in Table 2 represent instances of recall of study-list targets to their appropriate cues in the first stage of the three stages of the cued recall test, that is, recall of target items that the subject reproduced in response to the request to recall study-list items. 
Table 2

Recognition and Recall Proportions From the Critical Lists in the Present Experiment and in Reder et al. (1974, Experiment 2)

\begin{tabular}{|c|c|c|c|c|c|c|c|c|}
\hline & \multirow{3}{*}{$\begin{array}{c}\text { Recognition } \\
\text { Hits } \\
\end{array}$} & \multirow{3}{*}{$\begin{array}{l}\text { Cued } \\
\text { Recall }\end{array}$} & \multicolumn{2}{|c|}{ Recognized } & \multicolumn{2}{|c|}{ Not Recognized } & \multirow{2}{*}{\multicolumn{2}{|c|}{$\begin{array}{l}\text { Recognition Failure } \\
\text { of Recallable Words } \\
\end{array}$}} \\
\hline & & & & & & Not & & \\
\hline & & & Recalled & Recalled & Recalled & Recalled & Observed & Predicted \\
\hline $\begin{array}{l}\text { Immediate Test } \\
\text { Delayed Test }\end{array}$ & $\begin{array}{l}.98 \\
.50\end{array}$ & $\begin{array}{l}.95 \\
.60\end{array}$ & $\begin{array}{l}.94 \\
.39\end{array}$ & $\begin{array}{l}.04 \\
.11\end{array}$ & $\begin{array}{l}.01 \\
.21\end{array}$ & $\begin{array}{l}.01 \\
.29\end{array}$ & $\begin{array}{l}.01 \\
.34\end{array}$ & $\begin{array}{l}.01 \\
.38\end{array}$ \\
\hline \multicolumn{9}{|l|}{ Reder et al. (1974) } \\
\hline $\begin{array}{l}\text { High Frequency } \\
\text { Low Frequency }\end{array}$ & $\begin{array}{l}.36 \\
.83 \\
\end{array}$ & $\begin{array}{l}.63 \\
.78\end{array}$ & $\begin{array}{l}.31 \\
.67\end{array}$ & $\begin{array}{l}.05 \\
.16\end{array}$ & $\begin{array}{l}.32 \\
.11\end{array}$ & $\begin{array}{l}.32 \\
.06\end{array}$ & $\begin{array}{l}.51 \\
.14\end{array}$ & $\begin{array}{l}.52 \\
.10\end{array}$ \\
\hline
\end{tabular}

There were a few instances of recall of study-list targets in the second stage of the cued recall test. These instances are not included in Table 2, although they will be mentioned and summarized in a subsequent analysis below.

Three general observations of interest are yielded by the data in Table 2 . First, both recognition and cued recall were very high in the immediate test, and a very large majority of items (.94) were both recalled and recognized. Because of the very high level of recognition, the proportion of items that were not recognized but subsequently recalled was necessarily very low. Second, in the delayed test, both recognition and cued recall levels were considerably attenuated, and recognition failure correspondingly higher: Thirty-four percent of all the words that the subjects were capable of recalling were not recognized. Third, considering not only the data from the two tests in the present experiment, but also the high-frequency and lowfrequency data from the Reder et al. (1974) experiment, we notice a clear and striking negative correlation between recognition hits and recognition-failure measures. In the immediate test of the present experiment, probability of recognition was .98 and recognition failure given recall, .01; with low-frequency words in the Reder et al. experiment, the two measures were .83 and .14 ; in the delayed test in this experiment, they were .51 and .34 , and the high-frequency targets in the Reder et al. experiment yielded scores of .36 and .51 . All of these data very closely fit the function relating overall recognition hits and recognition failure that describes the extant data from a number of experiments (Tulving \& Wiseman, 1975), as shown by the "predicted" figures in the last column.
The frequency of false positive responses in the recognition test was essentially zero in the immediate test (only one such response occurred in all of the recall protocols), and $1.7 \%$ in the delayed test (30 responses out of 1,728 possible). False positive responses in the cued recall test were tabulated along with other response categories in that test, and those data will be presented shortly.

\section{Recognition of Phrase Cues}

In the cued recall test subjects were provided with 72 phrase cues: 24 corresponding to the study-list targets, 24 to "recognition-list" targets, words that had served as lures in the recognition test, and 24 corresponding to "semantic memory" targets, words that the subjects had not seen in the experiment (Table 1). The very first thing that the subjects did in the cued recall test was to indicate their recognition of phrase cues. In the immediate test, recognition of study-list cue phrases was very high; only 18 phrases out of the total 576 were not recognized, making a hit rate of 95\%. Recognition hit rate for the cue phrases in the delayed test was $74 \%$. False positive responses again were infrequent: $.2 \%$ in the immediate test and $3.4 \%$ in the delayed.

Given the classification of cue phrases into those that subjects recognized and those that they did not, it is possible to cross-tabulate the data on recognition and cued recall of study-list targets into a 2 by 2 by 2 table, in which the categories are: (1) recognition or nonrecognition of phrase cues, (2) recognition or nonrecognition of target words, and (3) cued recall or nonrecall of target words. Raw frequencies of these data are presented in Table 3 . The pattern of data

Table 3

Frequencies of Study-List Targets Recognized and Recalled, Associated With Recognized and Unrecognized Cue Phrases

\begin{tabular}{|c|c|c|c|c|c|}
\hline \multirow[b]{2}{*}{ Condition } & \multicolumn{2}{|c|}{ Recognized } & \multicolumn{2}{|c|}{ Not Recognized } & \multirow[b]{2}{*}{ Total } \\
\hline & Recalled & Not Recalled & Recalled & Not Recalled & \\
\hline & \multicolumn{5}{|c|}{ Immediate Test } \\
\hline Recognized Cues & 542 & 8 & 7 & 1 & 558 \\
\hline \multirow[t]{2}{*}{ Unrecognized Cues } & 0 & 17 & 0 & 1 & 18 \\
\hline & \multicolumn{5}{|c|}{ Delayed Test } \\
\hline $\begin{array}{l}\text { Recognized Cues } \\
\text { Unrecognized Cues }\end{array}$ & $\begin{array}{r}226 \\
0\end{array}$ & $\begin{array}{l}19 \\
47\end{array}$ & $\begin{array}{r}119 \\
0\end{array}$ & $\begin{array}{r}60 \\
105\end{array}$ & $\begin{array}{l}424 \\
152\end{array}$ \\
\hline
\end{tabular}


Table 4

Proportions of Study-List Targets Recognized and Recalled as a Function of Recognition and Nonrecognition of Phrase Cues

\begin{tabular}{lcccccrrr} 
& \multicolumn{3}{c}{ Target } & & \multicolumn{2}{c}{ Target } \\
\cline { 2 - 3 } Phrase Cue & Recognized & Not Recognized & & Total & & Recalled & Not Recalled & Total \\
\hline Recognized & .43 & .31 & .74 & .60 & .14 & .74 \\
Not Recognized & .08 & .18 & .26 & .00 & .26 & .26 \\
Total & .51 & .49 & 1.00 & .60 & .40 & 1.00 \\
\hline
\end{tabular}

in the immediate test is not particularly enlightening because of ceiling effects: A large majority of the total 576 targets were recognized and recalled to cue phrases that were also recognized. In the delayed test the pattern is more informative. For instance, the data show that not once did subjects recall a study-list target to a cue that they did not recognize. On the other hand, recognition of target words associated with unrecognized phrase cues occurred $31 \%$ of the time that this kind of event was possible $(47 / 152)$. It is also noteworthy that when the recognition and recall of study-list targets are conditionalized on recognition of cue phrases, there is a striking asymmetry between items not recognized but recalled (119 cases) and items recognized but not recalled (19 cases). Because recall never occurs to unrecognized cues, this asymmetry is considerably attenuated when the data are pooled over both recognized and unrecognized cues (119 vs. 66).

The data tabulated in Table 3 can be used to construct three different 2 by 2 contingency tables, two of which are interesting, inasmuch as they describe what, in a certain sense, appears to be a paradoxical situation. These two contingency tables are shown in Table 4. The one on the left shows that of all the phrasetarget pairs $43 \%$ were such that both their individual components, the phrase cue and the target word, could be recognized separately (in the absence of the other component); $39 \%$ of the pairs were such that one but not the other component could be recognized; and the remaining $18 \%$ were such that neither component could be separately recognized. If we used recognition hits as the (most sensitive) index about information about components of pairs available in the memory store, then we would conclude from the data tabulated in the left part of Table 4 that the upper limit of available information about complete phrase-word pairs at the time of the delayed test in this experiment was $43 \%$, the proportion of cases where both components could be recognized. The remaining pairs were either partly or completely "disintegrated," in the sense that only one, or neither, of the two components was recognizable. This conclusion, however, appears to be paradoxically at variance with the data on the righthand side of Table 4, which show that $60 \%$ of target words could be successfully recalled to phrase cues, all of these instances being accounted for in terms of recall to recognized phrase cues. How can $60 \%$ of the pairs be recalled if information is available about only 43\% of the complete pairs? The paradox, however, is more apparent than real. The data in question represent simply another way of stating what is already known, namely, recognition failure of recallable target words. The "paradox" exists only if we incorrectly assume that an unrecognizable item in one context could not be recalled in another context.

\section{Recall of Various Categories of Targets}

The reader is again reminded that there were three categories of target words in the cued recall test: studylist targets, recognition-test targets, and semantic memory targets. These different categories of target words could have been recalled by the subjects at any of the three successive stages of the cued recall test. The three stages succeeded each other, and were differentiated in terms of instructions to subjects to (1) recall study-list target words, (2) recall other recognition-test words, and (3) guess at possible completions of phrase cues by retrieving fitting words from semantic memory.

Table 5 summarizes the data on recall of target words, cross-tabulated in terms of the "origin" of the target word and its reproduction by the subject in any of the three successive stages. A further classification of the data in Table 5 is by immediate and delayed test. The percentages are all to the base defined by the total set of items in a particular category. This means that in the second and third stages they are not conditionalized on the number of opportunities to make appropriate responses.

Table 5

Proportions of Target Words in Different Categories Produced to Cue Phrases in Three Successive Stages of the Cued Recall Test

\begin{tabular}{|c|c|c|c|c|c|c|}
\hline \multirow[b]{2}{*}{ Origin of Target Word } & \multicolumn{2}{|c|}{$\begin{array}{c}\text { Recall of Study- } \\
\text { List Targets }\end{array}$} & \multicolumn{2}{|c|}{$\begin{array}{c}\text { Recall of Recognition- } \\
\text { Test Lures } \\
\end{array}$} & \multicolumn{2}{|c|}{$\begin{array}{l}\text { Guessing From } \\
\text { Semantic Memory }\end{array}$} \\
\hline & Immediate & Delayed & Immediate & Delayed & Immediate & Delayed \\
\hline Study List and Recognition Test & .953 & .599 & .003 & .045 & .000 & .000 \\
\hline Recognition Test & .000 & .009 & .071 & .083 & .003 & .010 \\
\hline Semantic Memory & .000 & .000 & .041 & .017 & .057 & .036 \\
\hline
\end{tabular}


The following observations based on the data in Table 5 are of interest. First, no false recognition test or semantic memory targets were produced as study-list targets in the immediate test of recall, and in the delayed test subjects reproduced only $.9 \%$ of recognition-test items, thinking that they were members of the original study list. These data show that subjects either will not or cannot produce words to cues if they did not see the words in the study list, even though they may have seen these words elsewhere and even though the words may otherwise (semantically) fit the cue. Second, there were a few instances in which a subject had forgotten that he saw a word in the study list, but remembered the item from the recognition test and reproduced it when requested to do so, in the second stage of the recall test. The proportions were $.3 \%$ in the immediate test and $4.5 \%$ in the delayed test. If these figures are conditionalized on nonrecall of study-list targets in the first stage, they become $6.4 \%$ and $11.2 \%$, respectively. Third, subjects did remember a certain small proportion of recognition-test lure words, and paired them appropriately with the phrase cues when instructed to do so. The relevant scores, $7.1 \%$ and $8.3 \%$ in the immediate and delayed tests, respectively, are of the same order of magnitude as the conditionalized recall levels of study-list targets as recognition-test items, which were, as just mentioned, $6.4 \%$ and $11.2 \%$, and higher than the levels of intrusions from semantic memory, $4.1 \%$ and $1.7 \%$. Fourth, when instructed to guess possible completions of phrase cues from semantic memory, subjects were not entirely unsuccessful; they got $5.7 \%$ and $3.6 \%$ items correct in the immediate and delayed tests, respectively. Finally, although in the delayed test subjects had not produced $35 \%$ of study-list targets in the first two stages in the cued test, not a single one of the "forgotten" items in this category was produced as a "guess" from semantic memory. The same thing happened in the immediate test, but because of the small number of opportunities in that test, the observation is not as striking. We mention this last finding in passing; we will have nothing further to say about it.

\section{DISCUSSION}

The main finding of this experiment was that words with a single dictionary meaning are subject to recognition failure: Although information is available in the memory store about the membership of a word in a studied list, the word cannot be recognized in the absence of its list context. This finding has several theoretical implications that need to be discussed.

It may be helpful to begin with a distinction between the occurrence and the magnitude of recognition failure. Recognition failure is said to have occurred when subjects cannot recognize some words that they can recall. The occurrence of recognition failure need not be theoretically important. It could be dismissed simply as a manifestation of unreliability of measurement, random fluctuation of trace or response strength, a consequence of additional opportunities for learning afforded by the recognition test as such, or in terms of other similar theoretically uninteresting possibilities. This is why it is necessary to consider the magnitude of the phenomenon of recognition failure, rather than just the fact of its occurrence. Many possible uninteresting explanations of recognition failure fall by the wayside when the magnitude of the effect becomes large.

A measure of recognition failure that has been successfully used in previous experiments is provided by the conditional probability of recognition miss given recall, that is, the ratio between the joint probability of recognition miss and recall, and the overall probability of recall. Thus, $\mathrm{P}(\overline{\mathrm{Rn}} \mid \mathrm{Rc})=\mathrm{P}(\overline{\mathrm{Rn}}, \mathrm{Rc}) / \mathrm{P}(\mathrm{Rc})$, as suggested by Watkins and Tulving (1975), where $\mathrm{Rn}$ and $\mathrm{Rc}$ designate recognition and recall hits, respectively, and $\overline{\mathrm{Rn}}$ refers to recognition miss. It is a useful measure for at least three reasons: (1) It makes intuitive sense, inasmuch as it directly expresses the proportion of recallable words that cannot be recognized, (2) it is theoretically free to assume any value between zero and unity, regardless of values of $P(R n)$ and $P(R c)$, and (3) it behaves in a highly systematic fashion as a function of overall recognition hit rate.

This last fact is especially important. It was demonstrated by Tulving and Wiseman (1975), who pointed out that the relation between recognition failure and overall recognition from all then extant experiments could be described quite well by the following equation:

$$
\mathrm{P}(\overline{\mathrm{Rn}} \mid \mathrm{Rc})=\mathrm{P}(\mathrm{Rn})-.5\left[\mathrm{P}(\mathrm{Rn})-\mathrm{P}(\mathrm{Rn})^{2}\right] .
$$

The bivariate data points from different experiments defined by recognition failure and overall recognition hit rate adhere very closely to this function, regardless of the differences in the procedure and materials used. Methodological and material variables may produce large differences in overall recall and in overall recognition, but they do not affect greatly the relation between recognition and recognition failure of recallable words.

The function relating recognition failure to overall recognition given in Equation 1 is interesting, not because it describes a correlation between these two variables, but because the correlation it describes is surprisingly high. There are no a priori reasons why one should not find a moderate amount of recognition failure under conditions where recognition hit rate itself is moderate. For instance, consider a situation in which the overall recognition hit rate is .40 , the overall recall is the same $(.40)$, where the joint probability of recognition hit and recall is .32 , and where, therefore, the joint probability of recognition miss and recall is .08 . The standard measure of recognition failure here 
(the conditional probability of recognition miss given recall) is $.08 / .40=.20$. Looking at these figures from the point of view of any theory of memory-formal, intuitive, or otherwise-one should not at all be prevented from at least contemplating the possibility that some particular experiment would yield such a set of recall/recognition contingencies. The invariant relation between overall recognition and recognition failure expressed by Equation 1, however, precludes such a possibility, or at least makes it extremely unlikely.

So far no data have been obtained in which large deviations from the function described by Equation 1 have been observed. In addition to experiments considered by Tulving and Wiseman (1975), other experiments have produced data which, when expressed in the appropriate format, closely adhere to the function (Antognini, 1975; Rabinowitz, Mandler, \& Barsalou, in press; Salzberg, 1976; Wiseman \& Tulving, 1976; Humphreys, Note 1). It is important to note that the bivariate data points fall very close to the function, regardless of whether recall is higher than recognition or recognition is higher than recall. In a number of experiments, recognition has been found to be higher than recall (e.g., Antognini, 1975; Postman, 1975; Salzberg, 1976), but when recognition failure in these experiments is conditionalized on recall, the resulting data are indistinguishable from those derived from experiments in which the overall recall is higher than recognition. Since the theoretically interesting finding is recognition failure of recallable words (Tulving, 1974; Tulving \& Thomson, 1973), the simple comparison of recall and recognition measures is not always informative: Recall superiority is a sufficient but not a necessary condition for recognition failure of recallable words (Wiseman \& Tulving, 1976).

The recognition failure data reported in the present paper are practically indistinguishable from those predicted by the Tulving and Wiseman (1975) function, as shown by the last two columns in Table 2. The discrepancies between the observed and predicted data may be real, in the sense that they reflect the systematic effect of one or more variables defining a given experimental condition, but at least at our present stage of knowledge, the broad agreement between the observed and predicted data deserves more attention than the discrepancies. The identical observed and predicted data in the immediate test in the present experiment can also be regarded as representing more than a mere coincidence, despite the very high levels of recall and recognition, and the presence of possible ceiling effects. Thus, given the basic relation between recognition and recognition failure expressed in Equation 1, it would be parsimonious to view even small amounts of recognition failure as reflecting the same processes that underlie large amounts, provided that overall recognition level is high. Small amounts of recognition failure that are coupled with low levels of recognition, on the other hand, could be more appropriately thought of as attributable to some other processes.

With the preceding discussion of the relation between recognition and recognition failure as a background, we are now ready to consider the theoretical significance of the results of the present experiment.

First, the data rule out any limitation of recognition failure to words that occur frequently in the language, or words that have multiple meanings or semantic senses: Recognition failure does occur with words that have only a single meaning as defined by dictionary entries. Infrequent and single-meaning words may be recognized more readily than frequent or multiplemeaning words, and, because of the systematic relation between recognition failure and overall recognition, this difference in recognition is also manifested in the magnitude of recognition failure. Thus, with other conditions held constant, the magnitude of recognition failure is positively correlated with word frequency and perhaps number of semantic senses, as Reder et al. (1974) have shown. But the generality of the recognition-failure phenomenon as such is questioned neither by the Reder et al. data nor by the present results.

Second, the finding that recognition failure occurs with single-meaning words casts doubt on the usefulness of the semantic interpretation of encoding specificity as proposed by Reder et al. (1974). This interpretation is predicated on the assumption that the probability of recognition failure varies with the number of semantic senses that to-be-remembered words have in (permanent) memory. This assumption may be correct insofar as the number of target words' semantic senses are correlated with the overall level of recognition and thereby correlated with the extent of recognition failure. The assumption may be incorrect insofar as it implies the existence of some class of items that, by virtue of the fact that they can only take a single semantic sense, are not subject to recognition failure. The present data rule out single-meaning words as candidates for such a class, and the Reder et al. (1974) data, as tabulated in Table 2 in the present paper, rule out low-frequency words. Only if a set of items could be identified which, when used as to-be-remembered units in an episodic memory task, would not exhibit recognition failure, would the semantic interpretation of recognition failure be strengthened. As matters stand right now, however, the semantic interpretation in the form proposed by Reder et al. (1974) appears to be in need of revision.

One possible line of revision would be to assume that all verbal units and utterances have multiple semantic senses, and that their recall and recognition in a particular situation depend on the relation between senses that are encoded at the time of study and senses 
that are retrieved at the time of test. Such a revision of the theory, however, would make it difficult to distinguish from other ideas that have been mentioned as possible explanations of the encoding specificity phenomenon, such as the idea that words presented in the context of other words might acquire "specific semantic meanings" (Tulving \& Thomson, 1973), that the meaning of a word presented in a particular context is only one of a "family of potential meanings" (Anderson, Pichert, Goetz, Schallert, Stevens, \& Trollip, 1976), that "instantiation" of a concept is determined by the total event described by the utterance of which a given word is a part (Barclay, Bransford, Franks, McCarrell, \& Nitsch, 1974), or that a particular context leads to the selection of a particular subset of a word's semantic features (Kintsch, 1974; Pellegrino \& Salzberg, 1975). Such a revision of the semantic interpretation would also become indistinguishable from a theory according to which the occurrence of any item in a to-be-remembered list creates a unique and novel trace of the event in the episodic memory system (Watkins \& Tulving, 1975). As yet there does not seem to be anything in the extant data that would compel the acceptance of one of these ideas in preference to others.

Third, the present results have implications for Martin's (1975) suggestion that the phrase "recognition failure of recallable words" is a misnomer, since the words subjects fail to recognize in one context are "different words" in another context. Martin argued that the to-be-remembered words used by Tulving and Thomson (1973), words such as LIGHT, COLD, CHAIR, and so on, although not specifically selected to be such, in fact were homographs. When a word such as LIGHT was presented as a member of the pair "head-LIGHT," the argument proceeded, the subject would store its "automobile part" meaning; when later on, in the recognition test, the word LIGHT was tested in the context of its associate dark, its "level of illumination" meaning would be consulted. Since this meaning had not been encoded, a recognition miss would result; later the originally encoded meaning could be recalled in the original study context.

The description of the present data as showing recognition failure of recallable words could not be so readily dismissed on the grounds advocated by Martin (1975). It is difficult to see how any one of the words used in the experiment, words such as BEVERAGE, PARAKEET, or GASOLINE, could be claimed, on purely intuitive grounds, to be "different" in different contexts. If such a claim were to be made, it would imply that every word in the language, or at least every noun, is a homograph. Such a conclusion would render the concept of a homograph meaningless.

We prefer the interpretation that the target words in this experiment, like the target words in other recognition-failure experiments, were specifically encoded with respect to, and as an integral part of, their study contexts, and that the resulting memory traces were sometimes more readily accessible through retrieval information extracted from one type of cue, for instance the context phrase, than another, for instance the copy of the nominal target. The words that the subjects fail to recognize are the same words that they can recall. Recognition failure simply illustrates the fact that the effectiveness of a retrieval cue depends on the properties of the trace of the word-event rather than on the characteristics of the target word in semantic memory (Tulving \& Thomson, 1973).

Fourth, the results of this experiment show that it is possible to hold constant the nominal identity of target words and observe large variations in the level of recognition failure, by manipulating other experimental conditions, such as the length of the retention interval. It follows, therefore, that semantic properties of target words neither constitute a necessary condition for the occurrence of recognition failure nor determine its magnitude. This fact places a burden not only on explanations of encoding specificity in terms of meaning or number of semantic senses of target words, but also on all other hypotheses that regard phenomena of encoding specificity as direct reflections of properties and structure of semantic memory. Semantic memory may well affect encoding of to-be-remembered events at the time of study, and equally well affect the interpretation and utilization of retrieval information available at the time of test, thereby also affecting the probability of occurrence of recognition failure, but in playing such a role it does not have any special status, since other factors also are known to influence encoding and retrieval of episodic information.

Finally, it is worth noting that "forgetting" observed in this experiment, the parallel effects of reduced recognizability and increased recognition failure with a 7-day retention interval, can be regarded as reflecting the increased specificity of the memory trace. Initially a very large proportion of to-be-remembered words could be retrieved through both phrase cues and copy cues, but as forgetting proceeded, the proportion of words recoverable through both cues decreased, while the proportion of words recoverable through only one cue increased. These observations corroborate similar ones made by Tulving and Watkins (1975) in an experiment in which the effectiveness of two extralist cues was measured over a retention interval, and similar increased specificity of the memory trace observed. Recognition failure is a special manifestation of the specificity of the trace, observed in a situation in which one of the retrieval cues is a copy cue.

The second purpose of the experiment was to examine the effects of a recognition test on subsequent recall of target words. Previous experiments that have dealt with this issue (e.g., Postman, 1975; Wiseman \& Tulving, 1975, 1976) have looked at the general effect of an interpolated recognition test on the recall test, 
and the effect of a target item's occurrence in the recognition test on its subsequent recall. In the present experiment, the focus was the subject's ability and willingness to utilize recognition-test information in recalling the target words from the study list in response to different instructions for such utilization. One relevant finding was that subjects do remember and can reproduce to cues some recognition-test lures, but they do so to any noticeable extent only when specifically instructed to recall recognition-test words. When instructed to reproduce words from the study list, subjects recall very few words that have occurred only in the recognition test. This suggests that subjects can discriminate between what they have learned in the study list and what they remember from the recognition test, and that the latter information is not applied to the solution of the problem of recalling study-list items. Another relevant finding was that in the recall test for the recognition-test words, subjects produced unrecognized study-list words at about the same level or rate as they produced recognition-test lures. In this test, with the data combined over both 0 - and 7-day retention intervals, the probability of recall of an unrecognized study-list item was about the same (.074) as the probability of recall of recognition-test lures (.077). Because of possible item-selection artifacts, one must be cautious in interpreting this equality, but it does suggest that their earlier appearance in the study list does not confer upon unrecognized target items any obvious advantages over items that have appeared only in the recognition test. In general, the findings pertaining to the second purpose of the experiment suggest that whatever additional learning of unrecognized study-list items occurs in the (first) recognition test, it is unlikely to greatly inflate the level of performance in the second test, and that, therefore, the recognition failure of recallable words does not reflect an artifact of additional learning opportunities afforded by the first test.

In conclusion, the results of the experiment extend the generality of the phenomenon of recognition failure of recallable words by showing that, with the overall level of recognition held constant, single-meaning words show as much recognition failure as words with multiple meanings. They also show that the amount of recognition failure can be varied by manipulating variables that affect overall recognition, even when all semantic properties of to-be-remembered items are held constant, thus suggesting that preexperimental properties of to-be-remembered items and the structure of semantic memory neither constitute a necessary condition for the occurrence of the phenomenon of recognition failure nor determine the magnitude of the phenomenon. Recognition failure, like all other phenomena of episodic memory, is directly determined by the relation between retrieval and encoding conditions, and only secondarily by semantic memory. Explanations of phenomena of encoding specificity in terms of semantic properties of to-be-remembered words can at best be incomplete. More complete explanations of the phenomena must be sought in the specific encoding operations performed upon the to-beremembered items at input and in the interaction of these encoding operations with specific conditions that prevail at the time of the attempted retrieval. Finally, the results of the experiment do not provide any support for the hypothesis that two retention tests given in succession are artifactually responsible for producing a state of affairs where unrecognized words can be subsequently recalled.

\section{REFERENCE NOTE}

1. Humphreys, M. S. Comparing recognition and recall. Paper presented at the 17 th Annual Meeting of the Psychonomic Society, St. Louis, Missouri, November 1976.

\section{REFERENCES}

Anderson, R. C.. Pichert, J. W., Goetz, E. T., Schallert, D. L., Stevens, K. V., \& Trollip, S. R. Instantiation of general terms. Journal of Verbal Learning and Verbal Behavior, 1976, 15, 667-679.

ANTOGNINI. J. The role of extralist associate cues in retrieval from memory. Unpublished $\mathrm{PhD}$ dissertation, Yale University, 1975 .

BADDELEY, A. D. The psychology of memory. New York: Basic Books, 1976.

Barclay, J. R., Bransford, J. D., Franks, J. J., McCarrell, N. S., \& Nitsch, K. Comprehension and semantic flexibility. Journal of Verbal Learning and Verbal Behavior, 1974, 13, 471-481.

COOPER, A. J.. \& MONK, A. Learning for recall and learning for recognition. In J. Brown (Ed.), Recall and recognition. London: Wiley, 1976.

Kintsch, W. The representation of meaning in memory. Hillsdale, N.J: Eribaum, 1974.

MARTIN, E. Generation-recognition theory and the encoding specificity principle. Psychological Review, 1975, 82, $150-153$

Miller, G. A., \& Johnson-LaIRD, P. N. Language and perception. Cambridge, Mass: Belknap, 1976.

Pellegrino, J. W., \& Salzberg, P. M. Encoding specificity in cued recall and context recognition. Journal of Experimental Psychology: Human Learning and Memory, 1975, 1, 261-270.

Postman, L. Tests of the generality of the principle of encoding specificity, Memory \& Cognition, 1975, 3, 663-672.

Postman, L., Jenkins, W. O., \& Postman, D. L. An experimental comparison of active recall and recognition. American Journal of Psychology, 1948, 61, 511-519.

Rabinowitz, J. C., Mandler, G., \& Barsalou, L. W. Recognition failure: Another case of retrieval failure: Journal of Verbal Learning and Verbal Behavior, in press.

Reder, L. M., Anderson, J. R., \& BJork, R. A. A semantic interpretation of encoding specificity. Journal of Experimental Psychology, 1974, 102, 648-656.

Salzberg, P. M. On the generality of encoding specificity. Journal of Experimental Psychology: Human Learning and Memory, 1976, 2, 586-596.

SLAmeckA, N. J. Recall and recognition in listdiscrimination tasks as a function of the number of alternatives. Journal of Experimental Psychology, 1967, 74. $187-192$. 
Tulving, E. Recall and recognition of semantically encoded words. Journal of Experimental Psychology, 1974, 102, 778-787.

Tulving, E., \& Tномson, D. M. Encoding specificity and retrieval processes in episodic memory. Psychological Review, 1973, 80, 352-373.

Tulving, E., \& Watkins, M. J. Structure of memory traces. Psychological Review, 1975, 82, 261-275.

Tulving, E., \& Wiseman, S. Relation between recognition and recognition failure of recallable words. Bulletin of the Psychonomic Society, 1975, 6, 79-82.

Watkins, M. J., \& Tulving, E. Episodic memory: When recognition fails. Joumal of Experimental Psychology: General, 1975, 104, 5-29.

Wiseman, S., \& Tulving, E. A test of confusion theory of encoding specificity. Joumal of Verbal Learning and Verbal Behavior, 1975, 14, 370-381.
Wiseman, S., \& Tulving, E. Encoding specificity: Relation between recall superiority and recognition failure. Journal of Experimental Psychology: Human Learning and Memory, 1976, 2. 349-361.

\section{NOTE}

1. There are some discrepancies between the Reder et al. (1974) data as given in Table 2 and as summarized in the original paper. The data in Table 2 are based on our own analysis of the raw recall protocols provided by Lynne Reder; it was difficult to completely reconcile the results of our analysis with those reported in the original paper.

(Received for publication May 3, 1977; revision accepted June 27, 1977.) 